\title{
Miranda
}

Revue pluridisciplinaire du monde anglophone /

Multidisciplinary peer-reviewed journal on the English-

speaking world

$12 \mid 2016$

Mapping gender. Old images ; new figures

\section{Bob Meets Sam}

Rencontre autour de Robert Wilson

Charles Chemin, Romain Fohr, Frédéric Maurin, Stéphane Boitel, Emeline Jouve et Margaux Szuter

\section{OpenEdition}

\section{Journals}

Édition électronique

URL : http://journals.openedition.org/miranda/8314

DOI : 10.4000/miranda.8314

ISSN : 2108-6559

\section{Éditeur}

Université Toulouse - Jean Jaurès

\section{Référence électronique}

Charles Chemin, Romain Fohr, Frédéric Maurin, Stéphane Boitel, Emeline Jouve et Margaux Szuter, "Bob Meets Sam », Miranda [En ligne], 12 | 2016, mis en ligne le 29 février 2016, consulté le 16 février 2021. URL : http://journals.openedition.org/miranda/8314 ; DOI : https://doi.org/10.4000/miranda. 8314

Ce document a été généré automatiquement le 16 février 2021.

\section{cc) $(9)$}

Miranda is licensed under a Creative Commons Attribution-NonCommercial-NoDerivatives 4.0 International License. 


\title{
Bob Meets Sam
}

\author{
Rencontre autour de Robert Wilson
}

Charles Chemin, Romain Fohr, Frédéric Maurin, Stéphane Boitel, Emeline Jouve et Margaux Szuter

\section{Introduction}

1 A l'occasion de la venue du metteur en scène et plasticien Robert Wilson pour La Dernière Bande mis en scène et interprété par Wilson, le Théâtre Garonne et l'Axe 3 («Faire Désordre ») du Laboratoire des Cultures Anglo-Saxonnes (CAS; EA 801; Université Toulouse Jean-Jaurès) ont organisé une rencontre autour de la représentation de la pièce de Samuel Beckett et plus largement du travail de l'artiste américain. La rencontre s'est tenue au Théâtre Garonne le 21 février 2015. Elle était à destination d'un large public composé de spécialistes du théatre, amoureux de cet art, curieux.

2 Chercheur en esthétique scénographique, Romain Fohr (Université de Picardie Jules Verne) a accepté notre invitation à jouer le rôle de discutant afin d'initier un échange entre Frédéric Maurin, Charles Chemin et les spectateurs présents. Maître de conférences à l'Université Sorbonne Nouvelle-Paris 3, Frédéric Maurin est spécialiste de l'œuvre théatrale de Wilson. Charles Chemin nous a fait la surprise et l'honneur de parvenir à se libérer de ses fonctions d'assistant à la mise en scène sur La Dernière Bande pour s'associer à la discussion. ${ }^{1}$ Comédien, metteur en scène, Chemin a beaucoup travaillé avec Wilson en tant qu'acteur mais également collaborateur sur de nombreux spectacles.

Emeline Jouve 
[Photo 1]

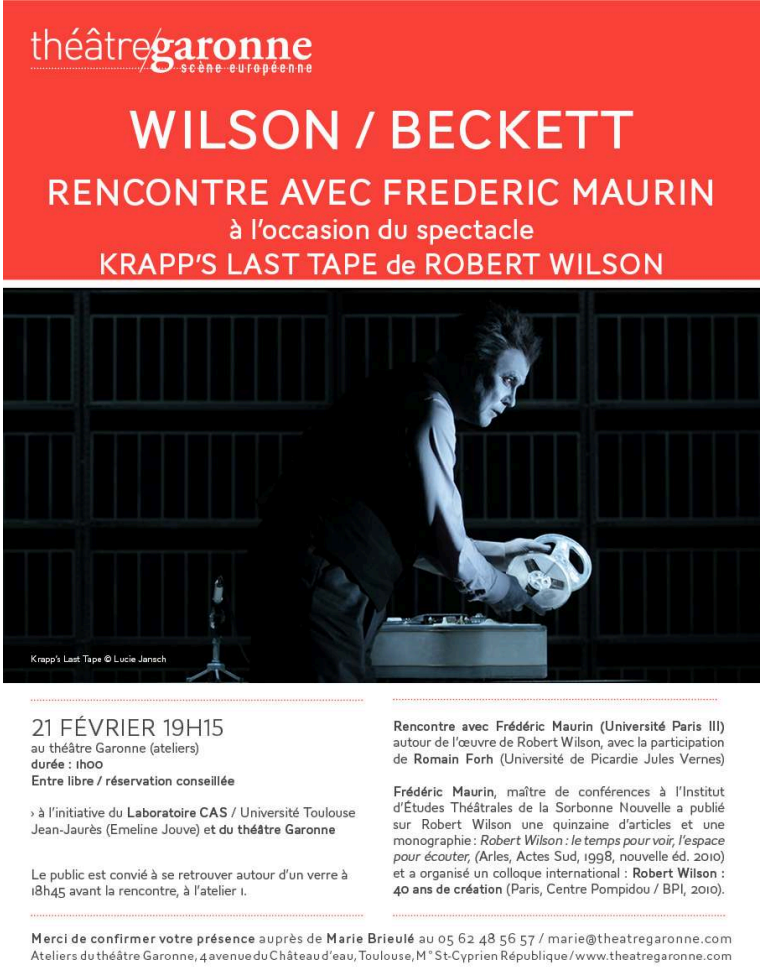

Flyer «Wilson/Beckett »

Crédit photo : Théâtre Garonne

\section{Biographie des intervenants}

4 Charles Chemin met en scène des spectacles entre théâtre, danse et arts visuels depuis 2008, notamment Girlmachine à la biennale Performa09 de New York ou PigPigPig à la biennale d'art contemporain de Moscou, et met en jeu les musiciens de l'ensemble Ictus dans Home Work du compositeur François Sarhan au Festival international de Bregenz. En 2015, il met en scène l'opéra Cubanacán de Roberto Valera à la Biennale de la Havane, repris en tournée en 16/17 aux Etats-Unis, et L'inventeur de l'amour d'après Gherasim Luca en 2016 au Théâtre national de Craiova en Roumanie. Son étroite collaboration avec Robert Wilson depuis 1993 le mène dans de nombreux pays, d'abord comme acteur puis assistant. Il est maintenant son co-metteur en scène pour les créations théâtrales, comme Garrincha à Sao Paulo, Les Nègres de Genet à l'Odéon, ou Rhinoceros de Ionesco, et participe à l'orientation artistique du Watermill Center, laboratoire de recherche fondé par Wilson à New York.

5 Frédéric Maurin est maître de conférences à l'Institut d'Études Théâtrales de l'Université Sorbonne Nouvelle-Paris 3. Ses recherches portent d'une part sur l'œuvre de metteurs en scène contemporains, et d'autre part sur la transversalité de questions esthétiques. Sur Robert Wilson, il a publié une quinzaine d'articles et une monographie (Robert Wilson : le temps pour voir, l'espace pour écouter, Arles, Actes Sud, 1998, nouvelle éd. 2010) et organisé un colloque international (Robert Wilson: 40 ans de création, Paris, Centre Pompidou / BPI, 2010). 
Romain Fohr est docteur en sciences humaines et sociales (Arts du spectacle Théâtre), enseignant-chercheur et membre du Centre de Recherche en Arts et Esthétique à l'Université de Picardie Jules Verne d'Amiens. Il collabore au groupe de recherches interuniversitaire et interdisciplinaire sur les revues de théâtre coordonné par Sophie Lucet (Paris7), Marco Consolini (Paris 3) et Romain Piana (Paris 3). Conférencier à l'école nationale supérieure des arts visuels de La Cambre Bruxelles. Enseignant au théâtre-école d'Aquitaine Pierre Debauche, à l'école nationale des arts du cirque de Rosny-Sous-Bois, au Pavillon Bosio de Monaco et au conservatoire d'Orléans. Il vient de publier Du décor à la scénographie. Anthologie commentée de textes sur l'espace scénique à L'entretemps.

\section{Transcription de la rencontre ${ }^{2}$}

7 ROMAIN FOHR: Merci beaucoup de votre accueil. Ça fait plaisir d'être dans une belle ville. Merci à Théâtre Garonne et au laboratoire CAS de Toulouse Jean-Jaurès de nous avoir réunis aujourd'hui. J'ai évidemment relu pour l'occasion l'ouvrage de Frédéric Maurin, Robert Wilson: le temps pour voir, l'espace pour écouter, et, comme je lui faisais remarquer tout à l'heure, il y a un lien fort entre Beckett et Wilson, puisque ce dernier a déjà mis en scène Oh les beaux jours en 2009. Il y a un point de convergence évident entre l'esthétique de Robert Wilson, son travail sur le son et la lumière, et ce qu'on a vu sur le texte proprement dit de Samuel Beckett. Celui-ci avait d'ailleurs assisté à un spectacle de Robert Wilson, lui avait parlé ensuite et lui avait confié tout le bien qu'il pensait de son travail. Robert Wilson y fait référence dans un entretien réalisé le 15 octobre $2013^{3}$ autour du spectacle que vous verrez ce soir. Dans ton ouvrage, Frédéric, j'ai relevé à six reprises des citations, des références entre Wilson et Beckett. Il y a une référence en particulier qui me parait extrêmement intéressante. Tu écris: "sauf à rappeler que le silence ni l'immobilité ne se peuvent concevoir sans le son et le mouvement dont ils sont la face cachée, ils demeurent sur le 'demi qui-vive' de Winnie dans Oh les beaux jours de Samuel Beckett $»^{4}$

8 FREDERIC MAURIN: Bonjour ! Avant d'entrer dans le vif du sujet, je voudrais tout d'abord remercier Émeline Jouve et Stéphane Boitel de m'avoir convié à cette rencontre, et de vous remercier aussi, chers auditeurs et spectateurs, d'être venus y assister. Ensuite, il me faut préciser que le livre que tu mentionnes, Romain, date de plus de quinze ans, bien qu'il ait effectivement fait l'objet d'une nouvelle édition en 2010. Lorsque j'ai écrit la phrase que tu as citée, et plus généralement esquissé la possibilité de parallèles entre l'esthétique de Robert Wilson et l'univers de Samuel Beckett, Wilson n'avait encore monté aucun texte de Beckett. Ces parallèles relevaient donc de l'analogie ; j'en avais une intuition un peu vague, mais il ne s'agissait en rien de rapprochements avérés : tout juste de convergences, peut-être d'affinités électives.

Or, il se trouve que d'autres convergences de ce type ont trouvé à se concrétiser au fil de la carrière de Robert Wilson. Si Gertrude Stein, par exemple, a nourri son travail dans les années 1970, s'il l'a invoquée pour étayer l'usage, « autiste » selon lui, qu'il faisait de la répétition dans les spectacles qu'il montait à l'époque avec un adolescent atteint de lésions cérébrales, Christopher Knowles, il a fallu attendre les années 1990 pour qu'il mette en scène des œuvres de Gertrude Stein à proprement parler : Quatre saints en trois actes, puis Saints and Singing. De même, l'intérêt de Wilson pour le théâtre japonais, en particulier pour le nô, est né d'une fascination de spectateur et de la 
sensation de travailler dans une direction voisine sous le rapport du temps, du formalisme, de l'artifice, de la stylisation; et ce n'est qu'ensuite qu'il a effectivement collaboré avec des artistes japonais comme Suzushi Hanayagi, assumé dans ses propres spectacles des emprunts au théâtre japonais, notamment dans Madame Butterfly de Puccini, puis monté, au milieu des années 1990, deux opéras tirés de pièces de nô : Hanjo et Hagoromo. Il n'en va pas autrement, d'ailleurs, pour le terme "opéra »: Aragon l'a employé le premier, faute d'une dénomination plus adéquate, pour qualifier Le Regard du sourd en 1971 (un "opéra sourd ", disait-il), Wilson l'a ensuite repris à son compte, en réactivant l'étymologie latine d'« œuvre » (opus), pour ranger sous cette appellation parapluie ses spectacles inclassables qui ne relevaient ni tout à fait du théâtre ni tout à fait de la danse. Mais c'est seulement en 1986 qu'il s'est tourné vers la mise en scène lyrique, vers le répertoire d'opéra au sens plein du terme : il a commencé par monter Alceste de Gluck, Salomé de Strauss, puis sont venus La Flûte enchantée, Parsifal et tant d'autres œuvres... Dans ces trois exemples, un même temps de latence sépare le sentiment diffus d'affinités et leur concrétisation effective, ou, en d'autres termes, l'intuition et le passage à l'acte, l'esprit et la lettre, l'ombre et la proie, le potentiel et le réel-comme on voudra. Ce temps, c'est le temps qu'il faut pour aborder l'horizon qu'on a juste entr'aperçu dans les brumes incertaines d'une possible communauté de vues.

Tout donne à penser que la rencontre avec le théâtre de Beckett a elle aussi été longtemps en germe avant de se matérialiser: non que Wilson ait toujours fait du Beckett sans le savoir, comme d'autres de la prose, mais son exploration du silence et de la durée, son travail sur la raréfaction du geste et la rétention du mouvement trouvent leur pendant (ou leur précédent?) dans l'amenuisement de la parole et l'appauvrissement du langage chez Beckett. Ionesco, au demeurant, l'avait déjà noté : Wilson et Beckett, disait-il en substance, partagent, chacun avec ses outils et ses visées propres, une même tendance à l'économie d'une part, ou au minimalisme, et à la dilatation d'autre part. Bien sûr, on aurait tort de céder aux vues rétrospectives ou aux réflexes émanatistes; mais il n'empêche que cette tendance commune suffit à justifier que Wilson décèle chez Beckett ce qu'on pourrait appeler un univers de correspondances. Dans cet univers, il se reconnaît presque comme dans un miroir, quelque légèrement déformé que puisse être le reflet renvoyé. Et il peut donc s'y projeter. Beckett représente pour lui une espèce de destin (ou du moins de destination), et le spectacle La Dernière Bande lui fournit opportunément l'occasion de rendre hommage à celui qui, comme l'a rappelé Romain, est venu féliciter, un soir de 1974, le jeune metteur en scène qu'il était alors. C'était après une représentation d'Une lettre pour la reine Victoria, spectacle que Wilson qualifiait d'« opéra de chambre » et qui était issu d'une étroite collaboration avec Christopher Knowles, que j'ai mentionné tout à l'heure : la logique et la rationalité étaient mises à mal, la parole défiait les lois de la langue, les sons s'atomisaient et se modulaient dans le reflux du sens, et c'est entre autres choses cette utilisation du langage qui avait beaucoup intéressé Beckett. En cela, La Dernière Bande est une espèce de boomerang, un hommage personnel en forme de dette. On y entend un écho, même lointain, du rapport au langage tel qu'il était exploité dans Une lettre pour la reine Victoria : Wilson, par exemple, parait savourer le mot, rare en anglais, viduity ("veuvage ») et il allonge à loisir le mot spool («bobine »); on dirait qu'il le mâche, qu'il le fait rouler en bouche, comme si la parole laissait place à un effet de chant, et la diction à un ersatz de vocalise. 
11 ROMAIN FOHR: Sur Wilson, j'ai découvert quelque chose qui m'a été soufflé par Frédéric quand on s'est vus il y a quelques semaines : je ne savais pas qu'il faisait des "portraits vidéo » animés et qu'il en avait notamment réalisé un de Winona Ryder prenant la pause de Winnie. Tu peux nous en parler un tout petit peu, parce que je pense que la salle ne connaît pas du tout cette œuvre-là?

FREDERIC MAURIN: Cette série de portraits a débuté il y a une dizaine d'années et il doit s'en compter une bonne cinquantaine aujourd'hui, tous dans des styles, des couleurs, des accompagnements sonores différents. Wilson fait appel à des célébrités, à des vedettes du théâtre, du cinéma, de la danse, du spectacle, et il les filme en citant le plus souvent des œuvres connues, des photographies ou des tableaux qu'ils animent d'un mouvement à peine perceptible. Johnny Depp, par exemple, recycle en couleur le travestissement de " Marcel Duchamp en Rrose Sélavy » de Man Ray, Isabelle Huppert se tient le visage entre les mains comme Greta Garbo dans le célèbre cliché de Cecil Beaton, Mikhaill Baryshnikov se surimprime à "Saint Sébastien » de Mantegna, et Lady Gaga, plus récemment, à «La Tête de saint Jean-Baptiste » de Solario, à « Mademoiselle Caroline Rivière » d'Ingres, à Marat dans «L'Assassinat de Marat » de David... Dans le portrait vidéo de Winona Ryder, on voit la tête de l'actrice émerger d'un mamelon comme le visage de Winnie dans la seconde partie d'Oh les beaux jours, avec, disposés devant elle, ces indispensables accessoires que sont le sac à main, le revolver et la brosse à dents du personnage de Beckett. Aucune parole, juste un morceau de violon composé par Michael Galasso ; aucun mouvement, sauf une légère inclinaison de la tête au bout de la douzaine de minutes que dure le portrait, comme l'amorce d'un baisser de rideau, comme le signe que le jour touche à sa fin - « le beau jour que ça aura été ! ». Or, ce jour, on l'a vu passer de l'aube au crépuscule dans le changement progressif de la lumière, dans le chatoiement sensuel des couleurs auquel se combine l'immobilité énigmatique de la figure muette. Certes, cette visibilité du passage du temps peut paraître paradoxale : d'une part elle est figurative (par opposition à l'abstraction des formes chère à Wilson), et d'autre part elle est plus rapide, dans sa représentation d'une douzaine de minutes, que la durée représentée (contrairement à l'élasticité et à la distension temporelles coutumières). Mais il n'en reste pas moins que la question du temps, qu'il coule ou s'englue, qu'il se replie sur lui-même ou vienne hanter le présent comme dans La Dernière Bande, ou encore qu'il paraisse s'absenter comme dans Fin de partie-la question du temps, donc, constitue une autre obsession commune à Wilson et Beckett. L'hommage chromatique contenu dans le portrait de Winona Ryder double, dans un langage de plasticien et de vidéaste, le travail du metteur en scène qui, Romain l'a dit tout à l'heure, a également monté Oh les beaux jours au théâtre, avec Adriana Asti dans le rôle de Winnie. Image contre langage, ce portrait consolide en acte les rapprochements potentiels entre l'épure visuelle et le monologue à mots comptés.

Pour en venir plus concrètement à La Dernière Bande, je vais maintenant me tourner vers Charles qui participe au spectacle depuis le tout début. Pourrais-tu nous expliquer comment l'idée est venue, comment vous avez travaillé ensemble?

CHARLES CHEMIN: Les spectacles de Bob se préparent souvent au Watermill Center, qui est un centre d'art que nous avons à côté de New York - à deux heures de Manhattan. Ils se préparent de manière très pauvre, sous des tentes, en plein soleil. Il peut faire jusqu'à 40 degrés, on n'a aucun moyen, on est assis à la table et Bob dessine. Tous les collaborateurs se rassemblent et on lui fait des propositions d'espaces, d'univers sonores et catera. Pour La Dernière Bande, il n'y avait pas encore l'idée d'en faire un 
spectacle quand on a débuté le projet ; en fait, ce devait être juste une petite lecture en marge de la création d'Oh les beaux jours à Luxembourg. J'ai commencé à travailler sous une tente avec le texte, à une table puis à m'amuser dans l'espace. Au bout d'une semaine, j'ai présenté une ébauche à Bob, mais il ne s'en est pas saisi tout de suite. Il était censé le retravailler par la suite, mais comme il avait des problèmes de santé à l'époque, ça ne s'est pas fait. On se disait pourtant que cette forme lui convenait très bien. Mais, comme il a un peu de mal avec les spectacles ou avec les textes dont on peut se dire qu'ils lui vont forcément très bien, ça a pris un peu de temps. On s'y est finalement mis pour le créer au festival de Spoleto en 2009. On est allés sur place et on a répété dans une salle assez miteuse, au sixième étage au-dessus du petit opéra à Spoleto, avec quasiment rien: une table, une chaise, un vieux bureau, aucun moyen normalement alloué aux créations de Bob. D'habitude, il y a des moyens dingues pour faire les premiers pas. Ici, au contraire, on n'avait pas d'ébauches de décor très élaborées, pas de sonorisation ou de micros. Ce spectacle s'est vraiment créé à partir de rien. Ensuite, au fil du travail, je suis monté sur le plateau parce que Bob a besoin de voir les choses : c'est quelqu'un de très visuel, il ne peut pas juste imaginer ce qu'il ferait comme acteur, il a besoin de diriger un autre acteur, comme en se dédoublant. J'étais donc sur le plateau, j'inventais, il regardait. J'ai aussi eu beaucoup plus de liberté que d'habitude, j'ai pu improviser-beaucoup-, ce qui, normalement, dans son travail, ne se fait pas vraiment. D'habitude il est très dirigiste, les acteurs obéissent à toutes ses indications depuis la salle : «bouge le bras dans telle direction », dit-il, ou « lève le petit doigt, » « tourne la tête », etc. Tout est minuté, tout est dirigé, en général, de manière presque dictatoriale. Là, en fait, il voulait voir évoluer quelqu'un de plus jeune-parce que je suis un peu plus jeune que lui-avec l'idée de se regarder, de s'écouter trente ans plus tôt, comme dans le texte de La Dernière Bande.

FREDERIC MAURIN: Petit à petit, au fil de ce travail en miroir, Wilson s'est donc luimême engagé dans le spectacle-un spectacle qui tourne depuis maintenant plus de cinq ans et qui, malgré des interruptions, a fait le tour du monde à raison d'une poignée de représentations par an. Cette longévité, ces reprises témoignent sans aucun doute que Wilson tient à ce spectacle : d'un point de vue artistique d'abord, puisque c'est pour lui l'occasion de jouer, mais peut-être aussi d'un point de vue personnel et, pour ainsi dire, affectif.

CHARLES CHEMIN: C'est un spectacle auquel il tient en effet beaucoup, il le propose souvent aux directeurs quand il est en création ou en tournée dans un pays, dans une ville, pour un autre spectacle. Cependant, il a quand même toujours peur de jouer parce qu'il n'a pas de formation d'acteur. Il a joué dans ses premiers spectacles, qui étaient foisonnants, souvent à base de n'importe quoi, où il délirait sur le plateau. Ensuite, il a joué en solo Hamlet : A Monologue, et maintenant La Dernière Bande. Ce sont ses seuls "spectacles d'acteur" et cela fait très peu pour un acteur; il appréhende donc beaucoup de se remettre sur un plateau, de se remettre en question. Mais il est très attaché à La Dernière Bande : il sent cet univers lui coller, il se donne aussi plus de liberté de jeu qu'il n'en accorde normalement aux acteurs. Ce spectacle-et, avec lui, l'univers de Beckett-a tourné dans de nombreux endroits et dans des cultures complètement différentes : en Corée, en Chine, beaucoup en Amérique du Sud, pas mal en Europe de l'Est ou en Russie. Chaque fois, c'est un vrai choc culturel. Bob a aussi un côté iconique, et comme ses grosses productions ne viennent pas forcément dans ces pays-là, cette forme assez pauvre, avec juste trois murs et des fenêtres, fournit en quelque sorte une porte d'entrée, une introduction à son travail. L'économie de moyens est très différente 
de la majorité des autres spectacles : il n'y a pas de cyclorama ni de poursuites. C'est un travail sur des choses vraiment très anciennes de son théâtre, des choses qui correspondent beaucoup plus à son théâtre des années 1970-80.

ROMAIN FOHR: Cette volonté qu'a Bob Wilson de présenter son spectacle dans le monde entier est très importante: on perçoit beaucoup d'influences aussi bien orientales qu'occidentales dans le geste, dans le mouvement, dans la parole, dans la voix. J'aimerais revenir sur le travail de répétition et cette liberté dont tu parlais. J'ai eu le plaisir de suivre les répétitions de Fables, d'après La Fontaine, à la Comédie-Française, spectacle dans lequel tu as joué, Charles. Il y avait une rigueur mathématique, rythmique, orchestré par Bob qui dirigeait avec des : « one step, two steps, three steps, number two, three steps... ». Je me souviens que c'était un peu désarçonnant pour Madeleine Marion, par exemple, qui était en fourmi et ne comprenait pas exactement tout ce qu'on lui disait. Mais dans La Dernière Bande, je dirais qu'on sent au contraire quelque chose de tellement libre...

CHARLES CHEMIN: Oui, mais cela tient au processus de travail. Personnellement, avec La Dernière Bande, j'ai eu une liberté que je n'avais jamais eue auparavant avec lui. Quand tu es venu voir ce que l'on avait fait sur les Fables à la Comédie-Française, tu as remarqué que chaque chose était mathématique, décidée, répétée et re-répétée dans son exact timing... Ici, on s'est plutôt attachés à travailler des attitudes, des ambiances, des atmosphères, des humeurs, des humeurs de jeu, des ressorts théâtraux que Bob n'utilise pas vraiment, en règle générale, ou alors qu'il établit avec une très grande précision pendant les répétitions sur d'autres spectacles. Ça tient au fait que j'étais sur le plateau et lui en face, et qu'ensuite on a renversé le processus: lui est passé au plateau, moi dans la salle, et la manière dont j'ai pu le diriger ne visait pas à supputer quelle était la chose juste à lui restituer ou la bonne formule; il s'agissait de lui réinsuffler mes impressions de l'intérieur, de retrouver le sentiment de l'acteur, pas celui du metteur en scène, puisque lui-même allait être acteur dans ce spectacle.

PUBLIC 1: J'ai eu la chance de voir le solo Hamlet il y a quelques années et j'aurais voulu savoir s'il y a des liens entre La Dernière Bande et Hamlet, qui est aussi un monologue. Car j'ai justement le souvenir d'avoir ressenti cette impression de grande liberté, ce que j'avais trouvé passionnant. D'où le plaisir, quelques années après, de revoir Wilson seul en scène.

CHARLES CHEMIN: En fait, ça dépend d'où et de quand vous avez vu Hamlet... Au départ, Bob s'était appliqué à lui-même les préceptes qu'il insuffle aux acteurs. Mais il s'est découvert aussi acteur et il s'est petit à petit libéré dans la forme. Au moment des représentations à Bobigny, il s'était déjà un petit peu libéré; ensuite, pendant la tournée, il s'est encore libéré davantage. Bob découvre aussi les choix dont l'acteur peut profiter : changer de petites choses de l'intérieur et tirer de la jouissance de ces changements.

21 FREDERIC MAURIN : À mon avis, Hamlet : A Monologue était un spectacle plus complexe que La Dernière Bande. Il commençait par la fin de la pièce de Shakespeare, au moment où Hamlet, sur le point de mourir après avoir été touché par Laërte, confie à Horatio le soin de raconter son histoire. Une fois les toutes dernières répliques prononcées, le spectacle remontait le fil de la pièce, en flash-back pour ainsi dire, ce qui n'est d'ailleurs pas sans rappeler la structure temporelle de La Dernière Bande; il se donnait donc pour le souvenir ou la réminiscence de la vie de Hamlet pendant son dernier souffle, pour son « re-membrement » en écho à l'injonction du spectre : « Remember ». Wilson jouait 
tour à tour les personnages principaux de la pièce, passait de l'un à l'autre, les faisait dialoguer, mais sans vraiment chercher à se travestir, notamment pour l'interprétation des rôles féminins de Gertrude et d'Ophélie qu'il considérait comme les deux piliers. Dans ce spectacle extrêmement structuré, il revisitait aussi la mémoire du théâtre, la variété des styles théâtraux, depuis des compositions plastiques très picturales jusqu'à des formes de cabaret populaire enjouées, des scènes de pantomime où on le voyait, pendant les interludes, apparaître vêtu d'un frac et coiffé d'un haut-de-forme devant le rideau tiré, ou encore un théâtre de marionnettes rudimentaire puisque c'est ainsi qu'il jouait la pantomime. Les affects perçaient plus que dans d'autres spectacles, on sentait un investissement personnel et autobiographique... sans doute un peu comme dans $L a$ Dernière Bande qui a à voir avec une jeunesse disparue. Dans La Dernière Bande, pourtant, ce n'est pas tant l'histoire du théâtre que Wilson me paraît revisiter, comme dans Hamlet, que l'histoire de son propre théâtre peut-être. Tu parlais tout à l'heure, Charles, de liens avec des spectacles des années 1970, et il me semble en effet que les rayonnages du fond de scène peuvent évoquer la bibliothèque d'I Was Sitting On My Patio, qui date de 1977, ou que les petits sauts au moment des saluts, s'ils assouplissent le formalisme parfois empesé de la représentation, s'inscrivent dans la continuité des bonds qu'il faisait et des rictus qu'il adressait aux spectateurs d'Une lettre pour la reine Victoria, qui date de 1974 et qui, encore une fois, avait tant marqué Beckett.

CHARLES CHEMIN: C'est tout à fait possible. Les saluts, au départ, ont été faits sans préméditation, mais maintenant on les travaille. Il fait ses petits sauts, effectivement, on établit un petit peu le parcours, ses demi-tours...

FREDERIC MAURIN: Si on peut voir, dans ces petits sauts, des clins d'œil en direction d'Une lettre pour la reine Victoria, c'est peut-être aussi parce que La Dernière Bande, plus généralement, permet d'établir, pour Wilson comme pour Krapp, un lien entre le présent et le passé. D'un côté, ces petits sauts peuvent paraître un peu empruntés (mais non pas au sens où ils seraient seulement empruntés au passé!), et de l'autre côté ils témoignent du plaisir du jeu, au double sens théâtral et ludique du terme, et renvoient donc, en un sens, à l'enfance et à ses pitreries. Cette tension me semble apporter au spectacle une note de tendresse très touchante. Au fond, que dit Wilson avec La Dernière Bande, sinon le consentement, serein et apaisé, au vieillissement, alors même que la jeunesse, pour douloureuse qu'elle ait été avec ses échecs, ses ruptures amoureuses, ses désillusions, demeure foncièrement présente, plus présente qu'un simple souvenir? Elle n'est ni oubliée ni reniée, encore moins enfuie ou enfouie, mais toujours susceptible de resurgir. Une homologie s'établit donc entre le personnage de Krapp et l'acteur Wilson. Est-ce d'ailleurs un hasard, un calcul ou une forme de providence si, au moment des premières représentations de $L a$ Dernière Bande, Wilson avait très exactement, comme on dit, l'âge du rôle : 69 ans ? C'est aussi pourquoi le spectacle ne se contente pas d'entrer en résonance avec Oh les beaux jours ou Hamlet: A Monologue. On peut également le rapprocher de Conférence sur rien de John Cage (Lecture on Nothing), que Wilson a notamment présenté lorsqu'il était le Grand invité du Louvre à l'automne 2013 à Paris. À nouveau un solo, à nouveau une résurgence du passé. Dans ce texte, qui date de 1949, Cage expose ses grands principes sur les valeurs a priori négatives du silence, de l'immobilité, du flottement ou de la vacance du sens, le tout ponctué de ce leitmotiv entêtant: "nous n'allons nulle part mais nous y allons lentement et sûrement. » Or, ce texte hante Wilson depuis longtemps : «j'ai lu Conférence sur rien et l'ouvrage Silence dans lequel il est inclus, dit-il, lorsque je suis arrivé à New York au début des années 1960, et j'en ai été marqué à jamais. » Cinquante ans plus tard, il le 
sort de sa bibliothèque personnelle, le monte et, ce faisant, s'expose lui-même. Toutes choses étant égales par ailleurs, le rapport de Wilson à Cage se solde par ce même hommage public que son rapport à Beckett avec La Dernière Bande : l'acquittement de la dette est identique, c'est de l'ordre d'un bilan ou d'un dévoilement de soi. Wilson offre au spectateur son passé d'acteur et les origines de sa formation artistique. Car en définitive, c'est lui qui est là, sur scène, qui nous regarde et nous en impose dans cette négociation avec lui-même. Je crois en effet qu'il est difficile de le dissocier du personnage qu'il joue, à tel point que le personnage paraît montrer Wilson plutôt que Wilson jouer le personnage et seulement le personnage.

CHARLES CHEMIN: C'est très juste. Il laisse transparaitre un peu de lui à travers le personnage de Krapp, mais aussi dans Conférence sur rien... En fait, on sent aussi ses aspirations, des choses qu'il répète depuis des années et des années et qui viennent effectivement de très loin.

FREDERIC MAURIN: C'est peut-être une des raisons pour lesquelles le solo est la forme dans laquelle il peut mettre le plus de lui-même, de son intimité, de ses affects, quitte précisément à ce que ses affects viennent amortir ou émousser les arêtes plus anguleuses du reste de son théâtre.

ROMAIN FOHR: Dans un article que tu as écrit il y a quelques années, Frédéric, tu as rappelé des propos de Wilson qui résument pour moi exactement ce dont on vient de parler : « dans mon théâtre, dit-il, on ne se donne pas à $100 \%$. Peut-être à 50 ou $60 \%$, de sorte que c'est moins appuyé [que le naturalisme]. $»^{5}$ Peux-tu développer ce point, parce que pour des gens qui font du théâtre, cela paraît absolument inconcevable, quand on voit ce qu'il fait, qu'il y ait cette marge de $50 \%$, justement, du temps, du silence, comme dans les 25 minutes au début de Krapp's Last Tape?

FREDERIC MAURIN: Ces propos de Wilson font écho aux principes du nô, dont le fondateur, Zeami, disait que l'acteur doit toujours être un peu en retrait, ne pas trop donner à voir ou à entendre pour permettre au spectateur de compléter ce qu'il voit et entend. L'acteur wilsonien n'extériorise pas tout non plus. Et c'est sans doute ce que veut dire Wilson, plus généralement, lorsqu'il souligne que le son continue dans le silence et que le silence est le prolongement du son, que l'immobilité est du mouvement, qu'elle est en mouvement, qu'il y a du mouvement en elle. Ce qui l'intéresse, c'est le surgissement de ce qui était invisible ou inaudible, mais qui était déjà présent, tout comme la disparition apparente de ce qui était visible ou audible, mais qui continue d'être présent dans l'invisible ou le silence. À charge pour le spectateur, donc, d'imaginer l'envers du spectacle, ce qu'il cache ou tait.

ROMAIN FOHR : Au cours des répétitions, quand choisissez-vous de vous arrêter pour fixer les choses?

CHARLES CHEMIN: En fait, pour une fois, on ne fixe pas tout. On conserve une marge, on conserve un "peut-être. » Donc " peut-être » qu'il va s'énerver davantage et avoir un rapport plus charnel avec la bande magnétique, avec la banane. «Peut-être » que le passage avec la banane va durer beaucoup plus longtemps, et il arrive d'ailleurs qu'il dure quelques minutes de plus que prévu dans certaines représentations. Bob s'octroie cette marge supplémentaire et joue ainsi avec ce qu'il a face à lui, quels spectateurs, mais aussi la configuration de la salle, sa position par rapport à cette salle, ce qu'il regarde... Et il réagit par rapport à ce monde-là. C'est quelque chose qui a toujours été très important dans son travail. Il y a aussi la part de chemin que fait aussi le spectateur 
vers lui. Et s'il maîtrise incroyablement bien ce rapport au public, c'est qu'il n'essaie pas de le forcer et qu'il en laisse un petit peu au hasard ; il réagit au moment présent.

FREDERIC MAURIN: Wilson a dit un jour, c'était dans les années 1970, qu'il rêvait d'un théâtre en continu, d'un théâtre qui se passerait par principe de spectateurs, où les gens seraient libres de venir, de rester quelques minutes, d'où ils partiraient et où ils reviendraient peut-être pour butiner et piocher ce qui les intéresse. Dans ce type de spectacle conçu à l'image d'un "supermarché »-la comparaison est de lui-, l'absence de logique narrative implique qu'on ne manque rien en s'absentant. En un sens, et c'est ce qui le rapproche de Gertrude Stein, Wilson n'a jamais voulu communiquer quoi que ce soit ni s'adresser frontalement au spectateur. Soit dit entre parenthèses, on lui a d'ailleurs reproché, en particulier lorsque le dogmatisme néo-brechtien avait force de loi en France, l'autisme de ses spectacles, leur absence de raison d'être en dehors d'euxmêmes. Dans leur esquive du politique et leur refus de livrer un discours, leur futilité a parfois paru décorative. Or, ce qui m'a énormément frappé ce soir, en voyant $L a$ Dernière Bande, c'est que ni le spectacle ni Wilson ne pouvaient se passer de spectateurs : j'ai eu l'impression qu'il lui fallait des témoins de ce qu'il dit, de ce que dit le personnage, de ce qu'il dit de lui à travers le personnage.

CHARLES CHEMIN: Et d'ailleurs il aguiche davantage le spectateur, il lui lance des clins d'œil, il lui fait des petits numéros de charme, et puis, hop, et il s'en écarte et reprend ses distances... Dans ce solo, il joue beaucoup plus avec ce rapport-là.

ROMAIN FOHR: Par rapport à l'écriture de Beckett, je retrouve beaucoup cela. Il y a cette jubilation à être avec le spectateur, à parler d'un moment si singulier et l'étirer, l'étirer, l'étirer autant que faire se peut. Peut-être que le spectacle de ce soir va durer une heure de plus...

CHARLES CHEMIN: Quand même pas une heure... Mais effectivement il y a un énorme différentiel dans ce spectacle. En général, d'une représentation à une autre, il peut $\mathrm{y}$ avoir 20 ou 30 secondes d'écart, quelquefois ça peut aller jusqu'à une minute, voire trois minutes dans le cas d'un spectacle très long. Avec La Dernière Bande, on a des différentiels, parfois, de 10 minutes, alors que c'est un spectacle court. C'est énorme! Effectivement, Bob va sentir et réinvestir un moment d'une manière complètement différente ce soir, peut être, par rapport à cet après-midi... Il faut revenir voir le spectacle... Car on peut réellement-et ce n'est pas de la démagogie de le dire-avoir un sentiment différent face à chaque représentation.

ROMAIN FOHR: Pour avoir vu La Dernière Bande hier et l'avoir revu cet après midi, je dirais honnêtement que l'écart était assez "foudroyant »-manière de parler, vu la foudre qui nous tombe sur la tête au début. Il y a un écart sensationnel et charmant avec, en effet, l'acteur qui est face à nous. C'est très troublant de se retrouver comme cela à deux mètres de lui, immobile. Il y a une telle connivence... Aujourd'hui, c'est rare au théâtre et c'est peut être une réponse à ce que tu disais, Frédéric, sur ce reproche qu'on a pu lui faire et qui n'était pas justifié.

FREDERIC MAURIN: Vaste question... On pourrait débattre longuement de ce reproche et de son éventuel manque de justification. Mais toujours est-il que le contexte des années 1970 était autre et que c'était peut-être plus insoutenable, pour toute une frange de spectateurs et de critiques européens, de voir un metteur en scène ne pas se préoccuper du sens et de proposer une aventure théâtrale purement sensorielle : d'où les accusations d'irresponsabilité politique, qui étaient le revers de la quête du beau. Quoi qu'il en soit, il me semble effectivement que lorsque Wilson est en scène, autre 
chose se passe. D'une certaine manière, il est le seul à pouvoir se permettre de déroger à son système et c'est ce que je trouve assez beau : au fond, autant on peut rêver d'un accident dans un spectacle de Wilson, autant c'est catastrophique lorsqu'il s'en produit un, sauf justement si c'est lui qui est en scène et qui le porte.

CHARLES CHEMIN: Oui. D'ailleurs, il y en a eu un cet après midi : un accident notable, même si vous ne l'avez sûrement pas vu. Sans tout dévoiler, ça a mis Bob dans un état autre ; il était beaucoup plus préoccupé par des choses liées au risque, au risque du pas suivant. Il était très perturbé et ce n'est pas forcément une mauvaise chose, en fait : car dans ces cas-là, il se ré-approprie le texte et il peut aussi en faire-mais tout dépend du point de vue-une force.

PUBLIC 4 : La saison étant aux rhumes, le public toussait beaucoup et j'avais l'impression que Wilson lançait des regards aux personnes qui toussaient... Est-ce que je me trompe?

CHARLES CHEMIN: Normalement, il ne supporte pas le bruit dans le public, c'est-à-dire qu'il a du mal si les spectateurs se manifestent. Au début où on jouait La Dernière Bande, il s'agaçait beaucoup en disant: " ils ne vont pas au théâtre en prenant des bonbons pour la toux ? C'est pas possible! » Petit à petit, en fait, il s'en est servi comme d'un paramètre de jeu. C'est très juste comme remarque. Oui, il peut avoir des regards de réaction au public...

ROMAIN FOHR: Il y a une interaction, mais je crois qu'il ne faut pas oublier qu'au théâtre, en particulier dans le théâtre shakespearien, il y a une interaction scène/salle très puissante et Wilson ne fait ni plus ni moins que rendre hommage de la plus belle des façons au personnage de Lear, du Choeur (Roméo et Juliette) et de Prospéro (La Tempête) pour n'en citer que trois exemples. Wilson nous dit : « vous existez dans cette sphère, dans ce monde que je recrée avec vous ». C'est vraiment troublant d'être avec lui avec ses petits clins d'œil, et ses petits sauts. J'ai une question sur ces danses magnifiques dans la pièce. On voit cet homme prostré avec ses pas du théâtre nô très glissés, puis tout d'un coup on le voit virevoltant, s'envolant, faisant des diagonales de danseur. Tu peux nous en parler, Charles?

CHARLES CHEMIN: C'est directement lié au fait de répéter avec quelqu'un d'autre sur le plateau, quelqu'un de plus jeune en miroir ; c'est comme se regarder dans un miroir, plus jeune, avec des démarches, des réactions, des mouvements qu'on peut davantage assimiler à un corps jeune. C'est tout cela que me suis attaché à lui retransmettre. Et la danse est venue de "bêtises ", j'ai un peu "improvisé » (entre guillemets) et fait "n'importe quoi ». Du coup, c'est indéniablement mon corps que je vois quand Bob joue. Il a travaillé, je l'ai aidé à acquérir une légèreté dans la marche, son pas n'est jamais lourd. Même s'il n'est plus tout jeune et qu'il n'a plus le corps de ses vingt ans, il déploie une grâce et une jeunesse stupéfiantes : c'est lié au processus qui consiste à regarder quelqu'un de plus jeune évoluer, mais aussi à fantasmer. Bob est quelqu'un de très obsessionnel, il fantasme beaucoup et rêve de celui qu'il était il y a quelques années. En cela, il est très lié au personnage de Krapp. Je ne sais pas si vous avez remarqué le maquillage, mais il montre le désir de revenir à une structure de visage plus jeune, d'avoir l'agilité, la grâce de quelqu'un de plus jeune. Si vous voyiez Bob marcher dans la rue, vous ne le soupçonneriez pas de pouvoir bouger comme il le fait sur le plateau. Il se dépasse. Même s'il a mal au genou, il arrive à en faire abstraction. équilibre entre conscience et oubli de soi. Par ailleurs, Wilson dit partager avec Beckett 
une fascination pour les grands comiques américains, en particulier Buster Keaton et Charlie Chaplin. Sauf projection fantasmée de ma part, il m'a semblé le voir adopter, à un moment dans le spectacle, la démarche de Charlie Chaplin, avec une main qui tiendrait la badine, comme il avait au reste demandé à Stefan Kurt, encore plus explicitement, de le faire pour interpréter le rôle de Mackie dans L'Opéra de quat'sous de Brecht au Berliner Ensemble.

CHARLES CHEMIN: ...Ça a été une grande influence pour le spectacle effectivement. On a regardé Buster Keaton et Chaplin et on avait même des photos dans la loge de maquillage pour refaire un peu les yeux et conserver quelque chose de ces comiques anciens.

ROMAIN FOHR: Frédéric, tu as suivi un grand nombre de spectacles de Wilson depuis des années, on peut en profiter puisqu'il nous reste cinq minutes pour évoquer le spectacle créé à l'Opéra de Paris et un second spectacle que tu as vu hier soir, sur la guerre de 1914. Peux-tu nous en toucher un mot ? Tout ça pour vous dire que quand Robert Wilson vient en France, généralement, il y a plusieurs spectacles qui se jouent simultanément et, évidemment, c'est aussi une organisation intellectuelle pour lui, je pense. C'est démentiel d'être à la fois comédien, metteur en scène à l'opéra, au théâtre, dans différentes villes en France et la semaine qui suit partir peut-être à Pékin ou à Ljubljana...

FREDERIC MAURIN: Ces deux spectacles sont de nature très différente-et c'est à mettre au crédit de Wilson que de proposer des créations qui, même si elles paraissent recycler certaines formules, comme lui en font grief ses détracteurs, relèvent de styles variés. Pelléas et Mélisande de Debussy a été créé en 1997 au Palais Garnier et vient d'être repris pour la sixième ou septième fois à l'Opéra-Bastille. D'une certaine manière, Wilson retrouve, dans le dépouillement de l'espace, dans l'éloignement des interprètes qui jamais ne se regardent, dans l'apparence de froideur et d'inexpressivité qui dissimule en réalité tout un travail sur la puissance des émotions, le rêve symboliste de désencombrer le plateau, voire d'en écarter l'être humain. La fameuse scène où Mélisande se coiffe en haut de la tour et où Pelléas caresse sa chevelure ondoyante en signe de passion érotique, est ici complètement biaisée : Pelléas se tient à plusieurs mètres de Mélisande qui se cambre en hauteur, et c'est précisément dans la tension spatiale que surgit un type d'émotion davantage plastique, qui n'a rien à voir avec l'émotion que pourrait susciter une représentation mimétique, illustrative, réaliste de cette œuvre qui, par essence, contrevient justement au réalisme. Plus encore qu'au théâtre, Wilson trouve à l'opéra le terrain où affirmer l'artificialité de la scène. Le second spectacle que tu évoques, Romain, a été créé à Prague il y a un an dans le cadre de la commémoration de la Première Guerre mondiale : il s'intitule 1914 et résulte d'un montage de deux textes, Les Derniers jours de l'humanité de Karl Kraus et Le Brave soldat Chvéik de Jaroslav Hasek. Après L'Affaire Makropoulos de Čapek, c'est le deuxième spectacle en tchèque que Wilson signe avec la troupe du Théâtre national (Národní Divadlo), comme il en a signé dans de nombreux pays et dans de nombreuses langues. On y retrouve l'actrice qui interprétait Emilia Marty, Soňa Červená, ex-diva des scènes lyriques née en 1925. Soit dit entre parenthèses, soulignera-t-on assez, un jour, l'amour incroyable que porte Wilson aux vieilles actrices? Bien avant Soňa Červená, Madeleine Renaud est apparue dans Ouverture en 1972, Marianne Hoppe a été «le » roi Lear à 81 ans en 1990 à Francfort, Inge Keller et Ruth Glöss (aujourd'hui décédée) du Berliner Ensemble ont respectivement incarné Shakespeare et le Fou dans Les Sonnets, à près de 
90 ans. Toujours est-il que 1914 est un spectacle musical qui raconte la fin de la Belle Époque, la fin des illusions, la fin de la croyance en l'impossibilité de la guerre, depuis les débordements initiaux d'une joie insouciante et apparemment intarissable jusqu'à la conscience désabusée qu'on peut encore vivre pire guerre que celle de 1914. On y retrouve aussi la veine du cabaret expressionniste qui innerve tout un pan de la création de Wilson depuis le début des années 1990, comme en témoignent récemment plusieurs spectacles réalisés avec le Berliner Ensemble qui ont fait le tour du monde.

ROMAIN FOHR: Madame Butterfly, un spectacle de 1993 entré au répertoire de l'Opéra de Paris, ouvrira la prochaine saison et va permettre d'amener du monde. Il faut rappeler que Wilson est aujourd'hui un des grands artistes mondiaux qui permettent de remplir les salles, on le voit ici, à Garonne.

FREDERIC MAURIN: Bob Wilson est un artiste, un metteur en scène, un vidéaste, un dessinateur, mais c'est aussi un manager, un businessman, le P.D.G. de la firme « RW Limited ». Le manège tourne. En l'espace d'une semaine, j'ai vu trois spectacles de lui sans même sortir de France.

PUBLIC 6 : Il ne faut pas oublier qu'il est nord-américain. Je n'arrive pas à penser à un autre metteur en scène qui tourne trois, quatre spectacles dans l'année...

ROMAIN FOHR: Il y a Robert Lepage en ce moment : c'est le même sauf qu'il est à Québec. Mais Wilson a, en effet, un opéra, un spectacle à Paris, un spectacle à Reims ... Robert Lepage et Robert Wilson se connaissent d'ailleurs et s'apprécient, je ne connais pas leur vie à tous les deux, mais je sais qu'ils s'apprécient.

FREDERIC MAURIN: Certes, Wilson est nord-américain, mais d'une part tous les nordaméricains ne sont pas aussi prolifiques, et d'autre part il représente en quelque sorte la figure de proue de l'internationalisation du théatre depuis les années 1980. On pourrait d'ailleurs se demander ce qu'il reste d'américain chez lui... Consciemment ou non, mais sans doute plus qu'aucun autre, il a très tôt joué la carte de la mondialisation artistique, du devenir planétaire ou cosmopolite de l'art. Mais à ce sujet, on pourrait aussi évoquer des metteurs en scène européens qui d'une part sont très prolifiques, et qui d'autre part, grâce au fonctionnement des théâtres de répertoire qu'ils dirigent, conservent et font tourner leurs spectacles pendant des années : je pense par exemple à Thomas Ostermeier, directeur de la Schaubühne de Berlin, et à Ivo van Hove, basé à Amsterdam, qui monte entre quatre et six créations par an, au théâtre ou à l'opéra, aux Pays-Bas et ailleurs, et qui reprend des créations plus anciennes.

Par ailleurs, Wilson vit dans l'impossibilité de s'arrêter, dans l'impossibilité de ne pas créer. Grâce au Watermill Center qu'a évoqué Charles tout à l'heure, il ne cesse de se projeter dans l'avenir et répète deux ou trois ans à l'avance ses prochaines productions. Cela tient du défi et d'un pari sur l'avenir, sans doute d'une angoisse de la disparition qu'il s'agirait de conjurer sans vouloir l'envisager, presque d'une assurance sur la vie. C'est entre autres raisons pour cela que La Dernière Bande me semble véhiculer quelque chose de touchant, dès lors qu'on y perçoit la volonté de continuer encore et toujoursmais sans nostalgie ni dissimulation du passé.

PUBLIC 7 : Une question: vous disiez qu'il allait reprendre Madame Butterfly... Savezvous s'il a l'intention de reprendre Woyzeck? C'est un spectacle que j'ai vu à Toulouse il y a une quinzaine d'années et qui m'avait fait l'effet d'une révélation. J'espère toujours qu'il va le rejouer... 

que Wilson reprenne un jour Woyzeck. La recréation d'Einstein on the Beach-la première a eu lieu en 2012 à Montpellier, les dernières représentations sont prévues en octobre prochain à Gwangju, en Corée du Sud-, cette recréation est l'exception qui confirme la règle. Quand on lui demandait d'en parler, Wilson disait : «Einstein, c'est le seul opéra, la seule œuvre du passé que je veuille remonter.» Il faut entendre dans ce remontage une remise en jeu, comme on le dit d'une somme qu'on a gagnée au casino, une remise en circulation, peut-être aussi une remise en question. Pour rappel, l'œuvre a été créée en 1976 au Festival d'Avignon, remontée pour quelques représentations à New York en 1984, reprise en tournée mondiale en 1992, avant d'être recréée, donc, avec des acteurs différents et des décors entièrement reconstruits en 2012. Mais Le Regard du sourd, le mythique Regard $d u$ sourd ne restera à jamais que dans le souvenir de quelques spectateurs et dans l'imaginaire de curieux qui ont consulté des écrits, des photographies, de rares images filmées. Pour ce qui est de Woyzeck et d'autres spectacles, c'est d'autant plus compliqué que, comme le Black Rider créé avec Tom Waits - premier spectacle à ouvrir la voie du cabaret dans laquelle Wilson s'est engouffré par la suite-, c'est un spectacle qui a été monté par d'autres que lui. Bien qu'il conserve son nom à l'affiche en tant que créateur de l'œuvre, la mise en scène a pu être revue in extenso par d'autres metteurs en scène. Einstein on the Beach l'a aussi été, c'est vrai : par Achim Freyer en 1988, dernier volet de la «trilogie » de Philip Glass après Satyagraha et Akhnaten. Mais il n'en reste pas moins qu'Einstein représente davantage, dans l'histoire des spectacles et la mémoire collective, l'œuvre de Wilson, une œuvre presque patrimoniale, un chef-d'œuvre artistique et culturel auquel ont pu assister différentes générations de spectateurs.

PUBLIC 7 : Je voulais dire qu'il y a des extraits du Regard du Sourd dans les archives de l'INA, je ne sais pas si tout le monde les a vus, c'est quand même assez intéressant de revoir ce spectacle de 1971.

voir des images de Wilson qui parle de lui-même, pour comprendre un peu d'où il vient, faute qu'on ait eu le temps d'en parler... Mais je trouve que le rapport au langage, par exemple, est aussi très important dans le lien entre Beckett et Wilson: ce serait un sujet intéressant à aborder. Enfin, c'est quelqu'un qui a eu un problème de langage dans sa jeunesse et qui a dû faire quelque chose contre ça, ou avec ça, et on ne peut s'empêcher de penser que ce qu'on voit ici-et dans toute sa carrière-en découle. Cette volonté de faire quelque chose contre ou avec le problème de langage, on la retrouve à mon avis dans La Dernière Bande transposée dans le corps, appliquée au corps: en tant que spectateur, j'ai eu la sensation, devant sa légèreté, qu'il allait ailleurs, qu'il faisait quelque chose de différent de l'endroit où il est d'habitude. Je pense qu'il y a quelque chose d'assez joli dans ce geste, comme tu disais tout à l'heure ; ce n'est pas vraiment testamentaire, je ne le dirais pas non plus comme ça, mais effectivement le regard dans le miroir est très impressionnant, à mon avis, tout comme la confusion entre l'acteur, ou l'icône qu'on a tous en tête, l'acteur qui est sur le plateau et le personnage de Beckett, si tant est que ce soit un personnage. La Dernière Bande est un spectacle très 
riche en fantômes, je trouve qu'ils sont nombreux sur le plateau avec Wilson, qui est tout seul en réalité.

Enfin, en écho à ce que tu viens de dire sur le fait que Robert Wilson est aussi un businessman, il faut rappeler que l'équipe qui l'accompagne est importante. A titre d'information-cela n'a rien de secret-, un solo de Wilson implique 12 personnes en tournée. C'est rare pour un solo qu'il y ait tant de personnes engagées. Je vous laisse donc imaginer ce que cela signifie pour des formes dites «lourdes». Garonne n'a programmé que quatre représentations, d'abord parce que le calendrier de Robert Wilson est très serré, ensuite parce que notre budget ne nous permettait pas d'en présenter beaucoup plus. On peut donc s'estimer déjà très chanceux, ce spectacle était un peu hors de notre portée, mais on a trouvé les moyens nécessaires pour se l'offrir. Pour vous l'offrir.

EMELINE JOUVE : Si Garonne a été très chanceux de pouvoir présenter Krapp's Last Tape, le public a lui aussi bénéficié de l'opportunité de non seulement voir un spectacle de Robert Wilson avec Robert Wilson mais également de pouvoir échanger aujourd'hui avec vous, Charles Chemin, Frédéric Maurin, Romain Fohr. Un grand merci à nos trois invités et au public au nom du Théâtre Garonne et du Laboratoire CAS de l'Université Toulouse Jean-Jaurès. Merci à tous.

\section{NOTES}

1. Charles Chemin n'est pas mentionné dans le programme initial intitulé «Wilson/Beckett: Rencontre avec Frédéric Maurin » car, en raison de son travail auprès de Robert Wilson qui jouait en amont et en aval de la rencontre, sa venue n'avait pas été envisagée au départ. Malgré son emploi du temps des plus chargés, Charles Chemin s'est généreusement libéré pour être des nôtres et nous lui en sommes particulièrement reconnaissants. Nous le remercions pour sa présence.

2. Nous souhaiterions remercier Margaux Szuter pour son travail de transcription.

3. "Automatic in the Muscle" : An Interview with Robert Wilson by Nicholas Johnson, 15 octobre 2013, Journal of Beckett Studies, vol. 23, nº 1 (2014): 101-106.

4. Frédéric Maurin, Robert Wilson : le temps pour voir, l'espace pour écouter, Arles : Actes Sud, 1998 : 86.

R. Fohr a présenté une contribution à l'Université de Reading qui précise les liens possibles entre Beckett et Wilson in Life Flashing in a Work of Art, Staging Beckett \& Contemporary Theatre \& Performance Cultures, University of Reading $11^{\text {th }}$ April 2015. Vous pouvez retrouver ce texte dans ce numéro d'Ariel's Corner.

5. Frédéric Maurin, « Nô comment : Robert Wilson et le théâtre japonais », Théâtre/Public, $\mathrm{n}^{\circ} 121$ (janvier- février 1995): 7-18. 


\section{RÉSUMÉS}

Transcription de la rencontre autour de la venue de Robert Wilson au Théâtre Garonne (Toulouse) le 21 février 2015. L'échange autour de la mise en scène par Wilson de La Dernière Bande fut organisé par le laboratoire CAS (Cultures Anglo-Saxonne) de Toulouse Jean-Jaurès (sur l'initiative d'Emeline Jouve) et le Théâtre Garonne (sur l'initiative de Stéphane Boitel) et réunissait trois intervenants en les personnes de Romain Fohr (discutant), Charles Chemin et Frédéric Maurin.

Transcription of the round-table on Robert Wilson's work at Théatre Garonne (Toulouse) on February, $21^{\text {th }}$ 2015. The discussion with Romain Fohr, Charles Chemin, Frédéric Maurin and the audience about Wilson's staging of Krapp's Last Tape was set up by the research group on the English-speaking world (CAS) of Toulouse Jean-Jaurès University and the Théâtre Garonne.

\section{INDEX}

Keywords : aesthetics, actor, American theater, Watermill Center, director, experimentation, monologue, Noh theater, opera, politics, portraits, spectator, strictness, tour, visual artist Mots-clés : Watermill Center, comédien, esthétique exigence, expérimentation, metteur en scène, monologue, portraits, spectateur, théâtre américain, théâtre nô, opéra, plasticien, politique, tournée

Thèmes : Theater

\section{AUTEURS}

\section{CHARLES CHEMIN}

Metteur en scène/Comédien

\section{ROMAIN FOHR}

Université de Picardie Jules Verne-Amiens

Docteur

\section{FRÉDÉRIC MAURIN}

Sorbonne Nouvelle-Paris 3

Maître de conférences

\section{STÉPHANE BOITEL}

Théâtre Garonne

\section{EMELINE JOUVE}

INU Champollion/Université Toulouse Jean-Jaurès

Maître de conférences

emeline.jouve@gmail.com 


\section{MARGAUX SZUTER}

Les Productions Parallèles

Manager d'artistes

margaux.szuter@gmail.com 